\title{
A Four Ingredients Chinese Herbal Formula, Decreases the Metastatic Recurrence of Postoperative Patients With Stage II- III Colorectal Cancer
}

Qi Shi ( $\nabla$ stevenshi_qi@hotmail.com )

Baoshan Branch, Shuguang Hospital https://orcid.org/0000-0001-8743-1134

Qiqi Le

Shanghai No.3 rehabilitation hospital

\section{Xing Wu}

Shanghai municipal hospital of traditional Chinese Medicine

\section{Xiyu Wang}

Shanghai municipal hospital of traditional Chinese medicine

\section{Minguang Zhang}

Shanghai municipal hospital of traditional Chinese medicine

\section{Fenggang Hou}

Shanghai Municipal hospital of traditional Chinese medicine

\section{Research}

Keywords: Chinese herbal formula, colorectal cancer, network pharmacology

Posted Date: May 4th, 2021

DOI: https://doi.org/10.21203/rs.3.rs-419697/v1

License: (c) (1) This work is licensed under a Creative Commons Attribution 4.0 International License.

Read Full License 


\section{Abstract}

\section{OBJECTIVE}

To identify the effect of traditional Chinese medicine (TCM) on postoperative patients with stage II-III colorectal cancer (CRC) and to explore the core herb combination and its mechanism.

\section{METHODS}

For this observational cohort study, patients diagnosed between January 2006 and September 2016 were enrolled from two hospitals, data was followed up from January 2019 to November 2020 and was analyzed in December 2020. The primary outcome, disease-free survival (DFS), was compared for those patients who received or did not receive TCM, and the secondary outcome was the hazard ratio (HR). The relevance principle was used to obtain the candidate herb combinations, and the core combination was evaluated through assessment of efficacy and representativeness. Then, the interactions among herbs and CRC were visualized by Cytoscape. Furthermore, related biological processes and signalling pathways were obtained by enrichment and KEGG analyses.

\section{RESULTS}

A total of 707 patients were finally included. In general, TCM decreased the metastatic recurrence associated with stage II-III CRC (HR: 0.61, log-rank P凶0.05). Among those patients in the TCM group, the core combination was Baizhu $\rightarrow$ Yinchen, Chenpi, and Fuling (C), and its antitumor mechanism was most likely related to 30 core targets, which were associated with 327 biological processes and 111 KEGG pathways. The most closely associated pathways were the PI3K-Akt, MAPK and HIF-1 pathways.

\section{CONCLUSIONS}

On the whole, a combination of four herbs, Baizhu $\rightarrow$ Yinchen, Chenpi, and Fuling, could reduce metastatic recurrence in postoperative patients with stage II-III CRC, and the mechanism might be related to the PI3K-Akt, MAPK and HIF-1 pathways.

\section{Introduction}

Colorectal cancer (CRC) is among the top three most malignant tumours in terms of new cases and deaths ${ }^{[1]}$. Although adjuvant chemotherapy and radiotherapy are available to treat postoperative CRC, nearly $35 \%$ of patients still develop metastatic recurrence ${ }^{[2]}$. In the Asian-Pacific region, especially in China, the incidence of CRC has also risen in recent decades ${ }^{[3,4]}$, and traditional Chinese medicine (TCM) has become a crucial adjuvant therapy for patients after radical resection.

The previous result of a retrospective cohort study suggested that TCM could reduce metastatic recurrence in stage I-III CRC patients ${ }^{[5]}$. However, not all postoperative CRC patients need to take medication; among them, patients with stage I CRC do not benefit from TCM. To date, the NCCN 
guidelines recommend chemotherapy as adjuvant therapy for patients with stage II-III disease, but at least $20 \%$ of patients develop metastatic recurrence ${ }^{[6,7]}$. The subgroup analysis in our above study indicated that TCM had the advantage of further reducing the metastatic recurrence of stage II-III CRC. However, TCM has specific characteristics, and the prescriptions of different attending doctors are not identical, so we do not know if an effective and representative drug combination exists among the prescriptions used for standardized treatment.

On the other hand, the oxygen-sensing pathway discovered by William G. Kaelin et al. is closely related to the prognosis of tumours, and HIF-1a is an important gene in this pathway ${ }^{[8]}$. Our previous study also found that Yi Ai Fang, a Chinese formula, can inhibit the invasion and metastasis of CRC through the regulation of HIF-1 ${ }^{[9]}$. However, whether the core drug combination among different prescriptions has the same effect or can act on other pathways simultaneously needs to be identified.

Therefore, we combined clinical data with bioinformatics and network pharmacology analyses to explore the core herbs of TCM in postoperative patients with stage II-III CRC and to identify the mechanisms that prevent metastatic recurrence.

\section{Methods}

Effect of Chinese herbs on stage II-III CRC

CRC patients enrolled from the Shanghai municipal hospital of traditional Chinese medicine, and the Baoshan Branch, Shuguang Hospital Affiliated to Shanghai University of Traditional Chinese Medicine met the following inclusion criteria: a) had stage II-III disease; b) were at least 18 years old; c) were diagnosed between January 2006 and September 2016; d) did not have synchronous metastasis; e) did not have any other primary tumour; $\mathrm{f}$ ) participated in follow-up every six months. The exclusion criteria are shown in Fig. 1. All patients provided written informed consent prior to follow-up. Research ethics boards approved the registered protocol (ChiCTR-00C-16009441).

The included patients were divided into two different groups according to whether they received or did not receive systematic TCM therapy. The definition of "systematic" was continuous treatment with Chinese herbs for at least six months. Of note, treatment was performed twice daily, and $200 \mathrm{ml}$ was administered each time in the TCM group in this study. The dosage of each herb was decided by the attending physician. In terms of clinical symptoms, the combination of herbs was adjusted within 1 or 2 weeks.

The primary outcome disease-free survival (DFS) was defined as the time from resection to relapse or death from any cause, whichever occurred first, and the hazard ratio (HR) was the secondary outcome if DFS did not attach. Moreover, propensity score matching (PSM) was used to correct for differences between groups.

Identification of the core combination 
Complete prescription information for patients in the TCM group was first collected, and the usage of herbs was recorded in Excel in the form of binary variables. After that, the underlying relevance of the prescriptions was analysed with a priori model (support $\geq 10 \%$, confidence $\geq 80 \%$ ) by using SPSS modeler (Version 14.0) to mine the herb combinations. Third, we selected the candidate combinations according to the support and confidence values. Then, DFS and HR were compared among the control group, the TCM group and those patients using the candidate combinations.

Identification of the core combination needed to meet the following three criteria: a) the patients who were defined as the core group used the complete candidate combination; $b$ ) the DFS of the core group was significantly longer than that of the control group, or the HR of the core group was significantly lower than that of the control group; $c$ ) there was a lack of significant differences between the core group and the TCM group in terms of DFS.

Ingredients and targets of the core combination

The ingredients of core combination were identified through the Traditional Chinese Medicine Systems Pharmacology (TCMSP, http://ibts.hkbu.edu.hk/LSP/tcmsp.php/), the National Scientific Data Sharing Platform for Population and Health (http://cowork.cintcm.com/engine/) and the Traditional Chinese Medicine Database @ Taiwan (http://tcm.cmu.edu.tw/zh-tw/). Oral bioavailability (OB, $\geq 30 \%$ ) and druglikeness ( $\mathrm{DL}, \geq 0.18$ ) were used to screen the active components, and their corresponding targets were identified through the TCMSP database.

CRC-related targets in the core combination

"Colorectal cancer" or "colorectal neoplasms" was used to identify CRC-related targets through the Online Mendelian Inheritance in Man (http://omim.org/), Therapeutic Targets Database

(http://bidd.nus.edu.sg/group/cjttd/), Genetic Association Database

(http://geneticassociationdb.nih.gov/) and the Pharmacogenomics Knowledgebase

(http://www.pharmgkb.org/). Moreover, all of the above described herbs or CRC-related targets were matched according to their gene id (homo sapiens) through the UniProt knowledgebase (http://www.UniProt.org/).

The protein-protein interaction (PPI) networks of the two kinds of targets were visualized through STRING (Version 10.5, https://string-db.org/), and the two networks were merged in Cytoscape (Version 3.5.1) to identify the duplicated targets.

Enrichment analysis

The degree centrality (DC) of the targets in the merged networks was analysed in Cytoscape, and then the median DC was used to identify the most important targets. After screening, Gene Ontology (GO) and Kyoto Encyclopedia of Gene and Genomes (KEGG) analyses of the remaining targets were performed using DAVID Bioinformatics Resources (Version 6.8, https://david.ncifcrf.gov/) and Omicshare tools

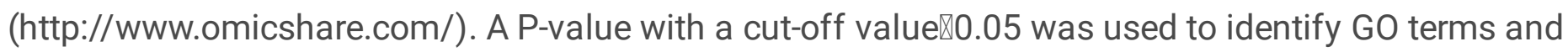


KEGG pathways. On the other hand, the ncbi-id and log FC values, which were retrieved from the Gene and Geo Datasets, respectively, from the National Centre for Biotechnology Information (https://www.ncbi.nlm.nih.gov/), were used to obtain the KEGG map. Biological processes and KEGG pathways were visualized through RGui 4.0.2.

\section{Statistical analysis}

Categorical and continuous variables were analysed by Pearson's $\chi^{2}$ test and Student's $t$ test, respectively. DFS was defined as the time from resection to relapse or death from any cause, whichever occurred first, and was evaluated with Kaplan-Meier curves and log-rank tests. Propensity scores were generated with logistic regression modelling to determine the probability of a patient undergoing TCM treatment based on gender, age, location, pathologic type, histodifferentiation, TNM stage, chemotherapy, radiotherapy and comorbidities. A 1:1 match with a random matching order and a 0.1 calliper was determined, and replacement was not allowed. All statistical analyses were performed using SPSS (version 24.0) and Stata (version 14.0). Hypothesis testing was two sided and conducted at the $5 \%$ level of significance. Data was followed up from January 2019 to November 2020 and was analyzed in December 2020.

\section{Results}

Effect of Chinese herbs on stage I-III CRC

A total of 707 patients were finally included (Fig. 1), of which 246 were in the control group and 461 were in the TCM group (Table 1). A total of 408 of 461 patients treated with TCM had adenocarcinoma compared with 197 of 246 control patients $(P=0.012)$; the difference in histology between the two groups was significant $(P=0.019)$. A total of 381 patients received adjuvant chemotherapy in the TCM group, and 185 patients received adjuvant chemotherapy in the control group $(P=0.001)$. After PSM, the baseline and tumour characteristics were not significantly different between the two groups (Supple Table 1). 
Table 1

Baseline and tumor characteristics of patients before propensity score matching.

\section{TCM group $(n=461) \quad$ Control group $(n=246) \quad P$ value}

age (y)

$₫ 60$

179

80

0.097

$\geq 60$

282

166

gender

male

248

136

0.705

female

213

110

location

colon

288

145

0.359

rectum

173

101

pathology

adenocarcinoma

408

197

$0.012^{\star}$

non-adenocarcinoma 2

unknown

51

2

histodifferentiation

poorly

50

29

$0.019^{*}$

moderately

291

127

well

11

2

known

109

88

TNM stage

\begin{tabular}{|c|c|c|c|}
\hline II & 225 & 137 & \multirow[t]{2}{*}{0.081} \\
\hline III & 236 & 109 & \\
\hline \multicolumn{4}{|c|}{ chemotherapy } \\
\hline yes & 381 & 185 & \multirow[t]{2}{*}{$0.023^{*}$} \\
\hline no & 80 & 61 & \\
\hline
\end{tabular}

* statistical difference 


\begin{tabular}{|llll|}
\hline & TCM group( $(\mathbf{n}$ 461) & Control group $(\mathbf{n = 2 4 6 )}$ & Pvalue \\
\hline yes & 37 & 19 & 0.887 \\
\hline no & 424 & 227 & \\
\hline comorbidities & & & $0.021^{*}$ \\
yes & 235 & 103 & \\
\hline no & 226 & 143 & \\
\hline * statistical difference & & & \\
\hline
\end{tabular}

The cumulative DFS after 1 to 6 years in the control group was $61 \%, 52 \%, 46 \%, 45 \%, 42 \%$ and $35 \%$. The cumulative DFS in the TCM group was $76 \%, 66 \%, 59 \%, 56 \%, 56 \%$ and $52 \%$. Before matching, the median DFS (mDFS) of the TCM group was not attached (Fig. 2A, HR: 0.61, log-rank P凶0.05). After matching, the risk of metastatic recurrence decreased in the TCM group (Fig. 2B, HR: 0.59, log-rank $P \bowtie 0.05$ ), although the mDFS was also not attached in the TCM group.

Identification of the core combination

Complete TCM prescriptions were retrieved for 314 of 461 patients and included 384 herbs. The results of the relevance analysis are shown in Supplemental Table 2. Among these, Fuling $\rightarrow$ Yinchen, Baizhu (A, Support: 29.53\%, Confidence: 87.14\%), Baizhu $\rightarrow$ Fuling, Huangqin (B, Support: 27.00\%, Confidence: 96.88\%), Baizhu $\rightarrow$ Yinchen, Chenpi, and Fuling (C, Support: 19.41\%, Confidence: 100\%) were the combinations with the highest support.

The risk of metastatic recurrence in patients treated with the $\mathrm{C}$ combination was significantly lower than that in the control group (Fig. 3A, HR: 0.43, log-rank $P=0.003$ ). In addition, patients treated with the $C$ combination were further compared with the TCM group, and there was no survival difference between these two groups (Fig. 3B, HR: 1.46, log-rank $P=0.184$ ).

Ingredients and targets of the core combination

There was a total of 45 ingredients in the herbs of the $\mathrm{C}$ combination based on OB and DL. Among these, 7 originated from Baizhu, 15 originated from Fuling, 10 originated from Chenpi, and 13 originated from Yinchen. Then, 216 targets associated with the above ingredients were matched. After being assessed in detail, 8 targets were excluded due to duplication. Finally, 208 ingredient-related targets were confirmed, and the PPI network is shown in Supplemental Fig. 1.

CRC-related targets of the core combination

CRC-related targets were retrieved from four databases: 81 from OMIM, 359 from GAD, 49 from TTD and 1058 from PharmGkb. After duplicate removal, 1076 targets were finally confirmed. 
Then, a merged PPI network of ingredients/CRC-related targets was generated, and the network indicated that there were $96 \mathrm{CRC}$-related targets in the core combination. These targets correspond to MOLO00049 of Baizhu, MOL000098, MOL000354 and MOL000358 of Yinchen, MOL000273 and MOL000296 of Fuling, and MOL004328, MOL005100, MOL005815 and MOL005828 of Chenpi (Fig. 4).

\section{Enrichment analysis}

Of the 96 targets, 30 were excluded due to a lack of corresponding reported pathways, and 36 were excluded due to their DC being under the average value of 14.6 , so 30 targets were finally subjected to GO and KEGG analysis.

There were 327 biological processes included, and they were mainly clustered in the following categories: 1) positive regulation of transcription from the RNA polymerase II promoter $(55.2 \%), 2$ ) positive regulation of transcription, DNA-templated $(51.7 \%), 3$ ) positive regulation of cell proliferation $(41.4 \%), 4)$ positive regulation of protein phosphorylation $(37.9 \%), 5)$ MAPK cascade $(37.9 \%), 6)$ negative regulation of apoptotic process $(37.9 \%), 7)$ signal transduction $(37.9 \%), 8)$ positive regulation of gene expression $(31.0 \%), 9)$ inflammatory response $(31.0 \%)$; and 10$)$ positive regulation of the ERK1 and ERK2 cascade (27.6\%) (Fig. 5A).

In addition, pathways involved in cancer was the highest term (75.9\%) among the $111 \mathrm{KEGG}$ pathways, and the enrichment proportions of the PI3K-Akt pathway (48.3\%), MAPK signalling pathway (44.8\%), and HIF-1 signalling pathway (37.9\%) were in decreasing order (Fig. 5B).

\section{Discussion}

Since the latest data show that the 5-year survival of stage I CRC patients is greater than $90 \%$, two recent studies in the Asia Pacific region have indicated that $15 \%-35 \%$ of patients with stage II-III CRC still have metastatic recurrence after receiving adjuvant chemotherapy ${ }^{[10,11]}$. Therefore, determination of ways to reduce the progression of stage II-III patients deserves more attention.

In China, Chinese herbal medicine is one of the most commonly used postoperative adjuvant therapies for CRC. Our previous studies suggested that although it could not further improve the DFS of stage I patients, it could reduce metastatic recurrence in stage II-III CRC ${ }^{[5]}$. However, the prescriptions given to 523 patients in the study involved a total of 384 herbs. It is still unknown which herbs can reduce the risk of postoperative metastatic recurrence. Therefore, we conducted this study.

Preliminary results showed that the results of this study were consistent with those of our earlier studies (PMID 28086238 and 29204061) ${ }^{[5,12]}$, indicating that TCM could reduce the risk of the metastatic recurrence of stage II-III CRC in general (HR: 0.59, log-rank Pख0.05).

To further explore the use of the core combinations in these patients, we first collected the prescriptions of 314 patients in the TCM group, and we finally identified three candidate combinations. However, 
among the three combinations, which is the core combination? One study from the Chinese Academy of Chinese Medical Sciences indicated that the results of prescription relevance analysis should be considered when examining combinations with high confidence and low support ${ }^{[13]}$. Therefore, we compared patients treated with the $\mathrm{C}$ combination to the control group and found that the risk for patients treated with the $\mathrm{C}$ combination was significantly lower than that for the control group $(P=0.003)$ and that there was no significant difference between patients treated with the $\mathrm{C}$ combination and the TCM group in terms of DFS $(P=0.164)$. Thus, we considered the $\mathrm{C}$ combination, Baizhu $\rightarrow$ Yinchen, Chenpi and Fuling, to be the core combination for prescription for patients with stage II-III disease, and its curative effect was representative. This may be related to the presence of atractylodes macrolide, tangerine peel, poria cocos polysaccharide and the extract of Yinchen because many studies have reported that the above components have antitumor effects ${ }^{[14-17]}$.

Since the clinical results suggested that the $\mathrm{C}$ combination could prevent the metastatic recurrence of stage II-III CRC, what was the potential mechanism of the $\mathrm{C}$ combination? One study from the Chinese Academy of Medical Sciences indicated that multiple components acting on disease-related targets were the basis of TCM ${ }^{[18]}$. In our study, 96 targets were associated with the $C$ combination, but 30 of these targets might play a more crucial role because of the increased DC. Therefore, we speculated that the $C$ combination might act through these 30 targets to prevent the metastatic recurrence of colorectal cancer most likely by involving the top 10 biological processes, including positive regulation of cell proliferation $(41.4 \%)$ and the negative regulation of the apoptotic process (37.9\%). This was similar to our earlier experiments, which indicated that Yi Ai Fang, a TCM formula, could participate in vasculogenic mimicry to inhibit CRC cell migration and invasion through HIF-1a ${ }^{[19-21]}$. In addition, the HIF gene has been confirmed to be associated with tumour angiogenesis and prognosis in hypoxia ${ }^{[22]}$. However, to determine whether HIF-related signalling pathways were relevant to the effect of the $\mathrm{C}$ combination, we undertook a further KEGG analysis and found that the top 3 most significant KEGG pathways among the 111 identified pathways were the PI3K-Akt (48.3\%), MAPK (44.8\%) and HIF-1 (37.9\%) signalling pathways. Our previous in vivo and in vitro experiments showed that EMT was regulated by HIF-1a to inhibit the invasion and metastasis of $C R C{ }^{[9]}$. However, how molecules downstream of HIF-1a regulate EMT is unknown. Interestingly, EGF, EGFR, MAPK1, MAPK3 and AKT1 were all involved in the above three pathways. According to the KEGG map, EGF and EGFR were shown to be upstream of the PI3K-Akt and MAPK signalling pathways (Supplemental Fig. 2). It was not clear whether TCM could play a multitargeting role by regulating the expression of EGFR and simultaneously affecting the two downstream pathways. In addition, cetuximab is a first-line drug targeting EGFR used for treatment of $\mathrm{CRC}$, but a number of studies suggested that differences in the primary sites of CRC were correlated with cetuximab efficacy ${ }^{[23-30]}$. The average PFS for left-sided CRC was 2.4 months longer than that for rightsided CRC. Therefore, whether the efficacy of the $\mathrm{C}$ combination is similar to that of cetuximab or whether there was a difference in efficacy due to the primary site needs to be confirmed by further data.

Strengths and limitations 
There have been an increasing number of studies on new TCM prescriptions utilizing big data mining, but few studies have conducted clinical re-evaluations of the mining results. This study evaluated the mining results in terms of validity and representativeness, and the clinical generalizability of this study is better than that of traditional prescription mining.

On the other hand, for stage II patients without high risk factors, the NCCN guidelines recommend followup or oral capecitabine. Due to the limitation of the sample size, this study did not conduct further subgroup analysis of patients with stage II and III CRC. Whether the drug combination is effective for all stage II patients remains to be further verified after the sample size is expanded. We speculate that this drug combination may inhibit the metastatic recurrence of CRC through the PI3K-AKT, MAPK and HIF-1 signalling pathways, but the specific mechanism still needs to be verified by experiments.

\section{Conclusions}

In general, TCM could reduce the metastatic recurrence of postoperative stage II-III CRC. Among the prescriptions used in CRC patients, Baizhu $\rightarrow$ Yinchen, Chenpi and Fuling was the core combination, and it could prevent metastatic recurrence. Moreover, this combination could inhibit the proliferation and migration of CRC, and we speculated that its effects are mostly associated with the PI3K-AKT, MAPK and HIF-1 signalling pathways.

\section{Abbreviations}

TCM: traditional Chinese medicine; CRC: colorectal cancer; DFS: disease-free survival; HR: hazard ratio; HIF: hypoxia inducible factor; PSM: propensity score matching; TCMSP: Traditional Chinese Medicine Systems Pharmacology; OB: Oral bioavailability; DL: drug-likeness; PPI: protein-protein interaction; DC: degree centrality; GO: Gene Ontology; KEGG: Kyoto Encyclopedia of Gene and Genomes; mDFS: median disease-free survival.

\section{Declarations}

\section{Acknowledgements}

Not applicable

\section{Authors' contributions}

Qi Shi, Minguang Zhang and Fenggang Hou designed, organized, and supervised the study. Qiqi Le drafted the manuscript. Xing Wu analyzed the literature. Xiyu Wang revised the manuscript. All authors read and approved the final manuscript.

\section{Funding}


Qi Shi's work on this paper was supported by the National Natural Science Foundation of China (NSFC81904005), the Scientific Research Foundation of Traditional Chinese Medicine of the Shanghai Health Bureau (20194Y0387), the Scientific Research Foundation of Traditional Chinese Medicine of the Shanghai Health Bureau (2018LP028) and the Scientific Research Foundation of Traditional Chinese Medicine of the Shanghai Health Bureau, ZY (2018-2020)-FWTX-6008.

\section{Availability of data and materials}

Not applicable.

\section{Ethics approval and consent to participate}

All patients provided written informed consent prior to follow-up. This study is registered with the Chinese Clinical Trial Registry (Trial Identifier, ChiCTR-O0C-16009441). Research ethics boards approved the registered protocol (2017SHL-KY-08).

\section{Consent for publication}

We declare that the Publisher has the Author's permission to publish the relevant Contribution.

\section{Competing interests}

The authors declare that they have no competing interests.

\section{References}

1. Siegel RL, Miller KD, Jemal A. Cancer statistics. 2020. CA Cancer J Clin. 2020. 70(1): 7-30.

2. Wilhelmsen $M$, Kring $T$, Jorgensen $L N$, et al. Determinants of recurrence after intended curative resection for colorectal cancer. Scand J Gastroenterol. 2014;49(12):1399-408.

3. Chen KQ, Li H, Sun KX, et al. Report of Cancer Incidence and Mortality in China, 2014. Chin J Oncol. 2018;40(01):5-13.

4. Feng RM, Zong YN, Cao SM, Xu RH. Current cancer situation in China: good or bad news from the 2018 Global Cancer Statistics. Cancer Commun (Lond). 2019;39(1):22.

5. Shi Q, Liu S, Li W, et al. Exploring the medication duration based on the effect of traditional Chinese medicine on postoperative stage I-III colorectal patients: a retrospective cohort study. Oncotarget. 2017;8(8):13488-95.

6. Böckelman C, Engelmann BE, Kaprio T, Hansen TF, Glimelius B. Risk of recurrence in patients with colon cancer stage II and III: a systematic review and meta-analysis of recent literature. Acta Oncol. 2015;54(1):5-16.

7. Quasar Collaborative Group. Gray R, Barnwell J, et al. Adjuvant chemotherapy versus observation in patients with colorectal cancer: a randomised study. Lancet. 2007. 370(9604): 2020-9. 
8. Kaelin WG Jr, Ratcliffe PJ, Semenza GL. Pathways for Oxygen Regulation and Homeostasis: The 2016 Albert Lasker Basic Medical Research Award. JAMA. 2016;316(12):1252-3.

9. Hou F, Li W, Shi Q, et al. Yi Ai Fang, a traditional Chinese herbal formula, impacts the vasculogenic mimicry formation of human colorectal cancer through HIF-1 $a$ and epithelial mesenchymal transition. BMC Complement Altern Med. 2016;16(1):428.

10. Benson AB 3rd, Schrag D, Somerfield MR, et al. American Society of Clinical Oncology recommendations on adjuvant chemotherapy for stage II colon cancer. J Clin Oncol. 2004;22(16):3408-19.

11. Yothers G, O'Connell MJ, Allegra CJ, et al. Oxaliplatin as adjuvant therapy for colon cancer: updated results of NSABP C-07 trial, including survival and subset analyses. J Clin Oncol. 2011;29(28):376874.

12. Liu SS, Shi Q, Li HJ, et al. Right- and left-sided colorectal cancers respond differently to traditional Chinese medicine. World J Gastroenterol. 2017;23(42):7618-25.

13. Li YB, Cui M, Yang Y, et al. Exploration the Similarities and Differences of Correlation Analysis and Frequency Statistics Methods of TCM Prescription. China Journal of Chinese Medicine. 2013;28(12):1940-3.

14. Xing KK, Tu YL, Chen SJ. Progress in Research on Anti-tumor Effect of Poria and cocos. Chongqing Journal of Research on Chinese Drugs and Herbs. 2019. (02): 45-49.

15. Wang HX, Huang HM, Ni FF, Cao CN, Zhao HP. Review of Antitumor Activities of Zangyinchen Extracts and Their Mechanisms. Chinese Journal of Ethnomedicine Ethnopharmacy. 2017;26(02):57-61.

16. Yang $X Z$, Zhang $H$, Cui $X Y$, et al. Study on the mechanism of nobiletin inhibiting the invasiveness of SGC - 7901 gastric cancer cells. Modern Oncology. 2020;28(18):3099-104.

17. Guo CX, Liu JB, Xie Q, et al. Atractylenolide â囚 inhibit the proliferation of gastric cancer cells SGC7901 by lowering Cyclin D1 / CDK4 and its mechanism. J Bengbu Med Coll. 2020;45(04):456-60.

18. Du GH, Wang YH, Zhang R, et al. Multi Component and Multi-target is the Surface Understanding of the Mechanism of Action of Traditional Chinese Medicine. World Science Technology. 2009;11(04):480-4.

19. Liu MM, Hou FG, Wu Q, et al. Experimental Research on "Yiai Decoction" in Treating Implanted Intestinal Cancer in Mice. SH J TCM. 2011;45(01):68-70.

20. Liu MM, Yu T, Chen M, et al. Effects of Yiaifang on Microvessel and Vasculogenic Mimicry Formation in Mice Bearing Transplanted Colon Cancer. Journal of Anhui TCM college. 2011;30(01):42-5.

21. Li W, Zong S, Shi Q, Li H, Xu J, Hou F. Hypoxia-induced vasculogenic mimicry formation in human colorectal cancer cells: Involvement of HIF-1a, Claudin-4, and E-cadherin and Vimentin. Sci Rep. 2016;6:37534.

22. Zong $\mathrm{S}$, Li W, Li H, et al. Identification of hypoxia-regulated angiogenic genes in colorectal cancer. Biochem Biophys Res Commun. 2017;493(1):461-7. 
23. Grothey A, Hedrick EE, Mass RD, et al. Response-independent survival benefit in metastatic colorectal cancer: a comparative analysis of N9741 and AVF2107. J Clin Oncol. 2008;26(2):183-9.

24. Elez E, Argilés G, Tabernero J. First-Line Treatment of Metastatic Colorectal Cancer: Interpreting FIRE3, PEAK, and CALGB/SWOG 80405. Curr Treat Options Oncol. 2015;16(11):52.

25. Stintzing S, Modest DP, Rossius $L$, et al. FOLFIRI plus cetuximab versus FOLFIRI plus bevacizumab for metastatic colorectal cancer (FIRE-3): a post-hoc analysis of tumour dynamics in the final RAS wild-type subgroup of this randomised open-label phase 3 trial. Lancet Oncol. 2016;17(10):1426-34.

26. Rivera F, Karthaus $M$, Hecht JR, et al. Final analysis of the randomised PEAK trial: overall survival and tumour responses during first-line treatment with mFOLFOX6 plus either panitumumab or bevacizumab in patients with metastatic colorectal carcinoma. Int J Colorectal Dis. 2017;32(8):1179-90.

27. Köhne $\mathrm{CH}$, Poston G, Folprecht G, et al. FOLFIRI plus cetuximab in patients with liver-limited or nonliver-limited RAS wild-type metastatic colorectal cancer: A retrospective subgroup analysis of the CRYSTAL study. Eur J Surg Oncol. 2016;42(10):1540-7.

28. Saito S, Hayashi N, Sato N, et al. Chemotherapy with bevacizumab for metastatic colorectal cancer: a retrospective review of 181 Japanese patients. Int J Clin Oncol. 2013;18(4):689-95.

29. Peeters M, Price T, Taieb J, et al. Relationships between tumour response and primary tumour location, and predictors of long-term survival, in patients with RAS wild-type metastatic colorectal cancer receiving first-line panitumumab therapy: retrospective analyses of the PRIME and PEAK clinical trials. Br J Cancer. 2018;119(3):303-12.

30. Wells JC, Tu D, Siu LL, et al. Outcomes of Older Patients ( $\geq 70$ Years) Treated With Targeted Therapy in Metastatic Chemorefractory Colorectal Cancer: Retrospective Analysis of NCIC CTG C0.17 and Co.20. Clin Colorectal Cancer. 2019;18(1):e140-9.

\section{Figures}




\section{From 2006 to 2016 ;}

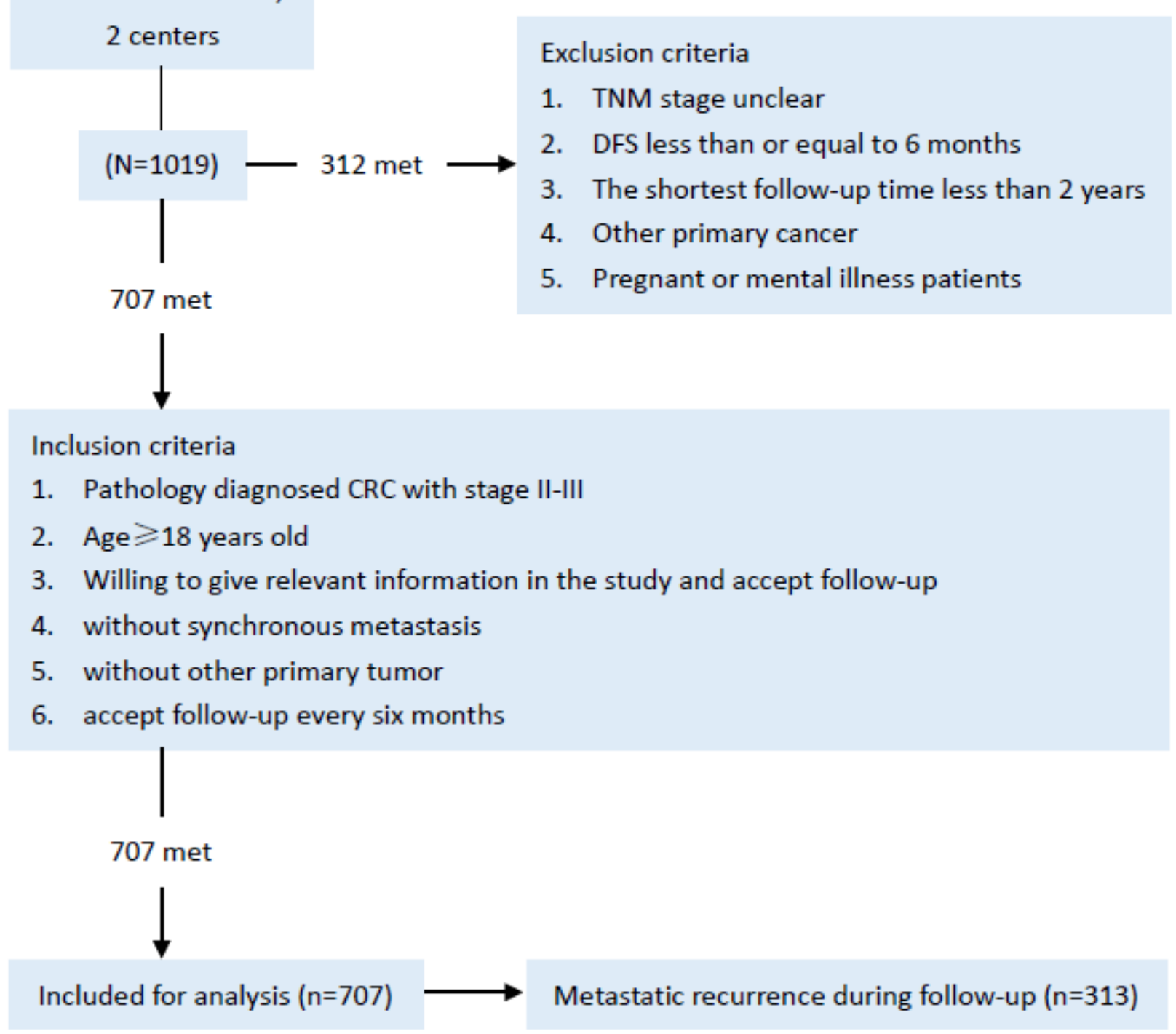

\section{Figure 1}

CRC patients enrolled from the Shanghai municipal hospital of traditional Chinese medicine, and the Baoshan Branch, Shuguang Hospital Affiliated to Shanghai University of Traditional Chinese Medicine met the following inclusion criteria: a) had stage II-III disease; b) were at least 18 years old; c) were diagnosed between January 2006 and September 2016; d) did not have synchronous metastasis; e) did not have any other primary tumour; $\mathrm{f}$ ) participated in follow-up every six months. The exclusion criteria are shown in Fig 1. All patients provided written informed consent prior to follow-up. Research ethics boards approved the registered protocol (ChiCTR-00C-16009441). 

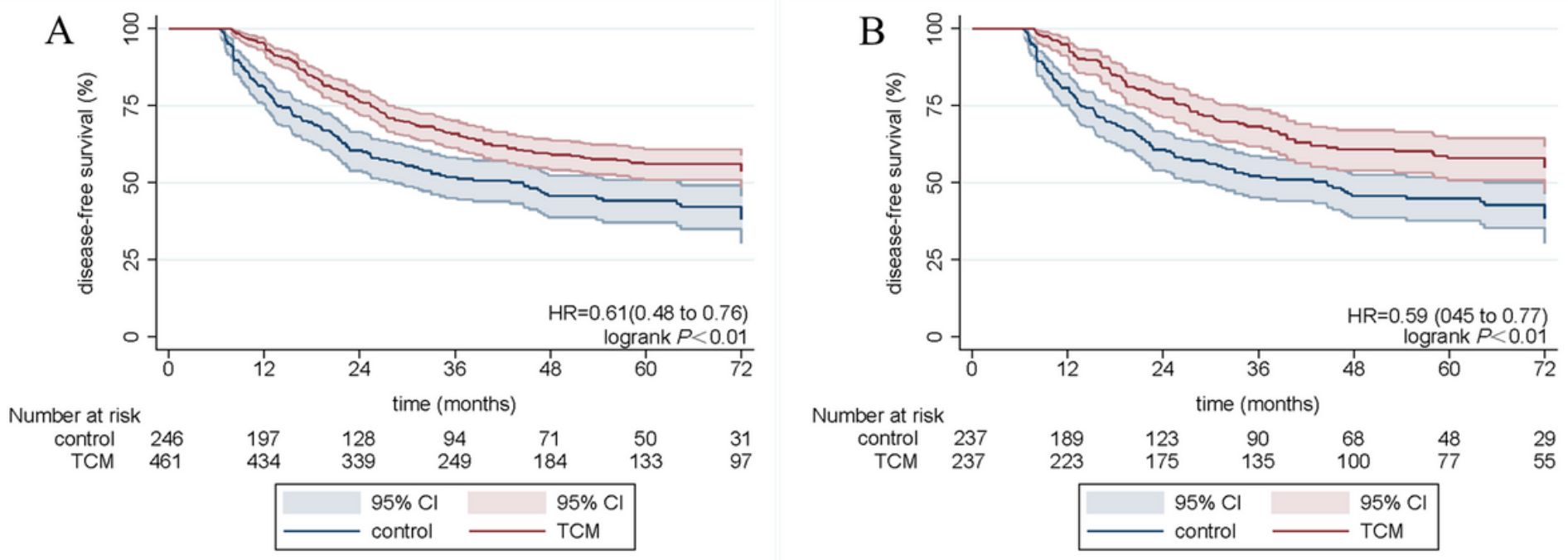

\section{Figure 2}

Effect of Chinese herbs on stage I-III CRC A total of 707 patients were finally included (Fig 1), of which 246 were in the control group and 461 were in the TCM group (Table 1). A total of 408 of 461 patients treated with TCM had adenocarcinoma compared with 197 of 246 control patients $(P=0.012)$; the difference in histology between the two groups was significant $(P=0.019)$. A total of 381 patients received adjuvant chemotherapy in the TCM group, and 185 patients received adjuvant chemotherapy in the control group $(P=0.001)$. After PSM, the baseline and tumour characteristics were not significantly different between the two groups (Supple Table 1). The cumulative DFS after 1 to 6 years in the control group was $61 \%, 52 \%, 46 \%, 45 \%, 42 \%$ and $35 \%$. The cumulative DFS in the TCM group was $76 \%, 66 \%, 59 \%$, $56 \%, 56 \%$ and $52 \%$. Before matching, the median DFS (mDFS) of the TCM group was not attached (Fig $2 A, H R: 0.61$, log-rank $P \otimes 0.05)$. After matching, the risk of metastatic recurrence decreased in the TCM group (Fig 2B, HR: 0.59, log-rank P囚0.05), although the mDFS was also not attached in the TCM group.
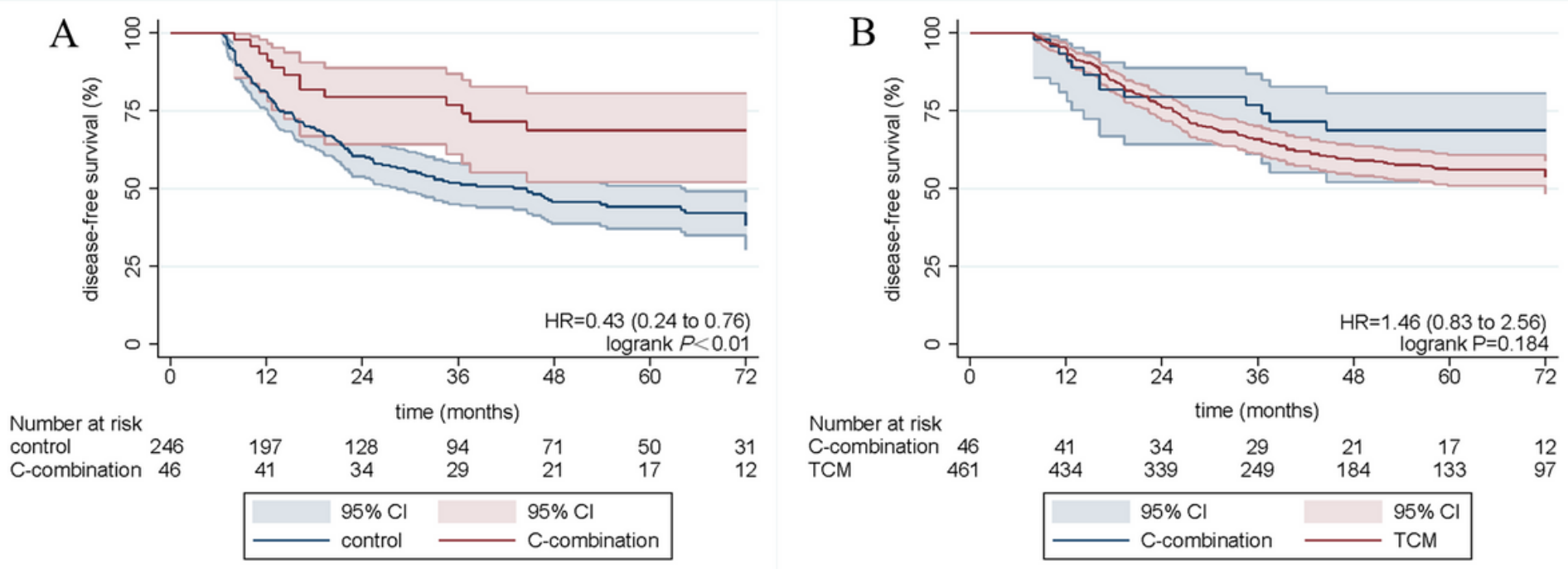

\section{Figure 3}


The risk of metastatic recurrence in patients treated with the $\mathrm{C}$ combination was significantly lower than that in the control group (Fig 3A, HR: 0.43, log-rank P=0.003). In addition, patients treated with the $C$ combination were further compared with the TCM group, and there was no survival difference between these two groups (Fig 3B, HR: 1.46, log-rank P=0.184).

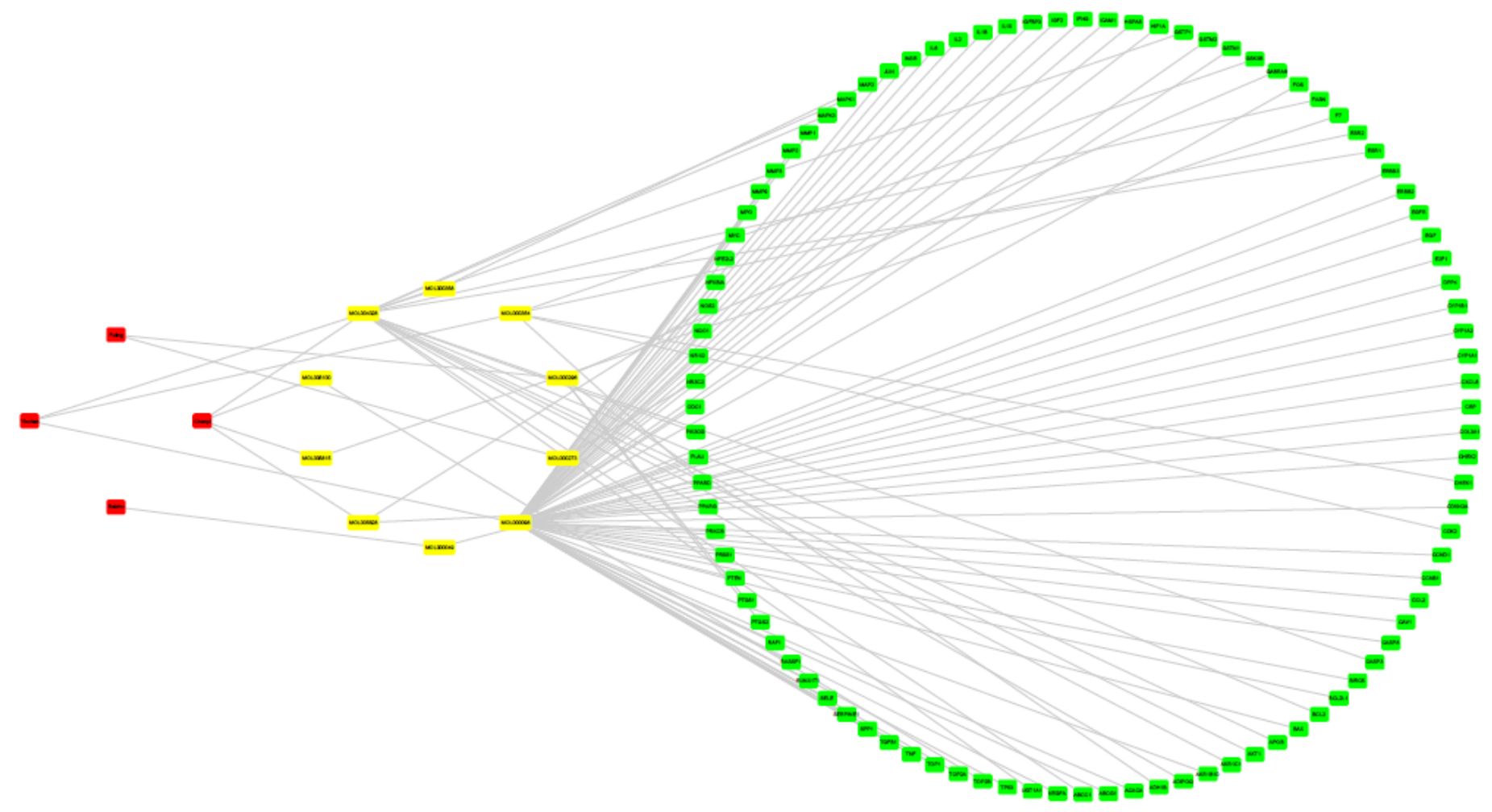

Figure 4

CRC-related targets of the core combination CRC-related targets were retrieved from four databases: 81 from OMIM, 359 from GAD, 49 from TTD and 1058 from PharmGkb. After duplicate removal, 1076 targets were finally confirmed. Then, a merged PPI network of ingredients/CRC-related targets was generated, and the network indicated that there were 96 CRC-related targets in the core combination. These targets correspond to MOL000049 of Baizhu, MOL000098, MOL000354 and MOL000358 of Yinchen, MOL000273 and MOL000296 of Fuling, and MOL004328, MOL005100, MOL005815 and MOL005828 of Chenpi (Fig 4). 


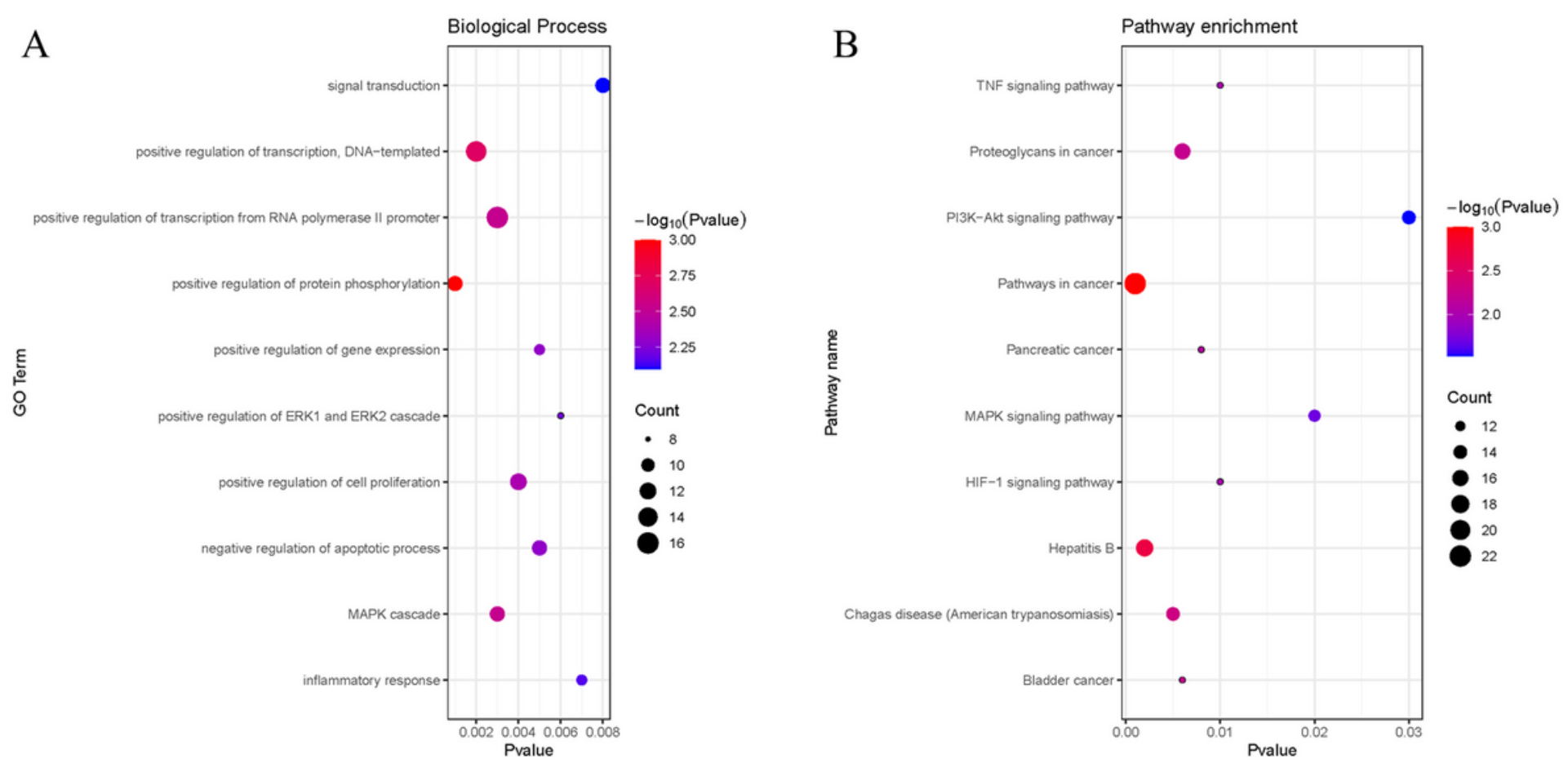

Figure 5

There were 327 biological processes included, and they were mainly clustered in the following categories: 1) positive regulation of transcription from the RNA polymerase II promoter $(55.2 \%), 2)$ positive regulation of transcription, DNA-templated $(51.7 \%), 3)$ positive regulation of cell proliferation $(41.4 \%), 4)$ positive regulation of protein phosphorylation $(37.9 \%), 5)$ MAPK cascade $(37.9 \%), 6)$ negative regulation of apoptotic process $(37.9 \%), 7)$ signal transduction $(37.9 \%), 8)$ positive regulation of gene expression $(31.0 \%), 9)$ inflammatory response $(31.0 \%)$; and 10 ) positive regulation of the ERK1 and ERK2 cascade (27.6\%) (Fig 5A). In addition, pathways involved in cancer was the highest term (75.9\%) among the 111 KEGG pathways, and the enrichment proportions of the PI3K-Akt pathway (48.3\%), MAPK signalling pathway (44.8\%), and HIF-1 signalling pathway (37.9\%) were in decreasing order (Fig 5B).

\section{Supplementary Files}

This is a list of supplementary files associated with this preprint. Click to download.

- Supplementarytable1.docx

- Supplementarytable2.docx 